\title{
Exercise promotes heart regeneration in aged rats by increasing regenerative factors in myocardial tissue
}

\author{
A. ESKANDARI ${ }^{1}$, R. SOORI ${ }^{1 *}$ ๑, S. CHOOBINEH ${ }^{1}$ and Z. MAZAHERI TIRANI ${ }^{2}$ \\ ${ }^{1}$ Department of Exercise Physiology, Faculty of Physical Education and Sport Sciences, University of \\ Tehran, Tehran, Iran \\ ${ }^{2}$ Basic Medical Science Research Center, Histogenotech Co., Tehran, Iran
}

Received: April 20, 2019 • Accepted: January 07, 2020

Published online: April 14, 2020

(C) 2020 Akadémiai Kiadó, Budapest

\begin{abstract}
Exercise-induced stem cell activation is implicated in cardiovascular regeneration. However, ageing limits the capacity of cellular and molecular remodelling of the heart. It has been shown that exercise improves structure regeneration and function in the process of ageing. Aged male Wistar rats $(n=24)$ were divided into three groups: Control (CO), High-intensity interval training (HIIT) $(80-100 \%$ of the maximum speed), and continuous endurance training (CET) (60-70\% of the maximum speed) groups. Training groups were trained for 6 weeks. The expression of the Nkx2.5 gene was determined by real-time (RT-PCRs) analysis. Immunohistochemical staining was performed to assess the C-kit positive cardiac progenitor and Ki67 positive cells. The mRNA level of Nkx2.5 was significantly increased in the CET and HIIT groups $(P<0.05)$. Also, cardiac progenitor cells positive for $\mathrm{C}$-kit were increased in both the CET and HIIT groups $(P<0.05)$. Exercise training improved the ejection fraction and fractional shortening in both training groups $(P<0.05)$. This study indicated that training initiates the activation of cardiac progenitor cells, leading to the generation of new myocardial cells $(R=0.737, P=0.001)$. It seems that $\mathrm{C}$-kit positive cells in training groups showed an increase in the expression of some transcription factors ( $\mathrm{Nkx} 2.5$ gene), representing an increased regenerative capacity of cardiomyocytes during the training period. These findings suggest that the endogenous regenerative capacity of the adult heart, mediated by cardiac stem cells, would be increased in response to exercise.
\end{abstract}

\section{KEYWORDS}

cardiac stem cell, C-kit, Nkx2.5, exercise, ageing

\section{INTRODUCTION}

Ageing is a physiological phenomenon characterized by a gradual decrease in the ability of the replacement of old cells by new ones [18]. Studies have indicated that ageing induces functional

\footnotetext{
*Corresponding author. Tel.: +98 2188351741. E-mail: soori@ut.ac.ir
} 
and structural changes in the heart [16]. Biological ageing is associated with a progressive decrease in cardiac myositis as a result of age-related cardiac diseases [27]. There is evidence that indicates cardiomyocytes are able to regenerate in the human heart, but this process seems to be very slow and only a small number of cardiomyocytes can be replaced during a human lifespan [6, 9, 21].

Exercise training improves the health and performance of the cardiovascular system and denotes a potent tool for cardiovascular therapy. Exercise exerts its beneficial effects through reducing cardiovascular risk factors, and directly affecting the cellular and molecular remodelling of the heart [14].

Studies showed that cardiac stem cells (CSCs) play an essential role in the replacement of cardiac cells after heart failure. Although the underlying mechanisms of the dysfunction of CSCs in the ageing process are not fully understood, it has been shown that CSCs are decreased by ageing [5]. On the other hand, exercise training improves the decreased size of heart CSCs during the ageing process $[14,22]$. Therefore, exercise could induce beneficial effects for the regeneration of the aged heart. Exercise training enhances some biological functions in the myocardium, and leads to physiological hypertrophy and cellular and molecular remodeling [1,25]. In addition to heart physiological hypertrophy especially in cardiomyocytes, it has been shown that cardiac cell number is increased due to the division of pre-existing cardiomyocytes induced by exercise [7].

The CSCs include C-Kit+, Sca-1+, and cardiac muscle-derived stromal cells [13]. C-kit positive cells are well-known cells located in the human heart, and their activation leads to the formation of the new myocardium [10,23]. C-kit positive cells can be identified by particular phenotypic markers and possess clonogenic and self-renewal capability, as well as the capacity to differentiate into the three major cardiac lineages: myocytes, endothelial cells, and smooth muscle cells [13, 25].

Waring et al. showed that the adult heart responds to an increased workload with physiological hypertrophy, cardiac stem cell activation, and new myocyte formation [25].

Cardiac remodelling could be induced by many signalling pathways, such as the Notch and the Wnt pathways. These signalling pathways regulate the expression of transcription factors which are essential for the growth of the heart tissue [28]. Transcription factors for the development of the heart include GATA transcription factors and Nkx2.5. Nkx2.5 plays a critical role in the control of gene expression [20]. It has been reported that the amount of Nkx2.5 gradually decreases in the course of the ageing process. However, the molecular mechanisms underlying the exercise-induced improvement in the regeneration of the aged heart are mostly unclear. While training intensity can also be important in this regard, enhamcement of the adaptation of physical training, while minimizing the time and effort devoted to training, is a subject of considerable interest among exercise scientists [12]. Therefore it seems that intensive training might induce the differentiation of CSCs into cardiac cells, whereas the evidence about the effect of exercise on C-kit and $\mathrm{Nkx} 2.5$ expression in aged subjects is still very slim.

Therefore, in the present study, we hypothesized that high-intensity interval training (HIIT) and continuous endurance training (CET) are able to improve cardiac function and cardiac regeneration in aged rats.

\section{MATERIALS AND METHODS}

\section{Animal and ethical statement}

Twenty-four aged male Wistar rats (400-450 g, aged 22-24 months) were obtained from the Pasteur Institute of Iran and randomly divided into the three groups, control (CO), continuous endurance 
training (CET), and high-intensity training (HIIT) $(N=8$ per group). Rats were housed in cages under a controlled environment $\left(23{ }^{\circ} \mathrm{C}\right.$ and 12 -h light-dark cycle) with free access to normal chow and tap water. All experimental procedures were approved by the Ethics Committee of Tehran University of Medical Sciences, Tehran, Iran (IR.ut.Rec.1395005). CO animals maintain their own activity without exercise training during a 6-week period. The animals were familiarized with motorized treadmill running for two weeks. The individual maximal exercise capacity test was performed as the maximum speed reached by each animal. In the CET group, rats ran for 5 min ( 5 days per week for 6 weeks) at $40-50 \%$ of the maximum running speed (warm-up), 65\% of the maximum speed for $15 \mathrm{~min}$ (first week), 65\% of the maximum speed for $20 \mathrm{~min}$ (second week), 70\% of the maximum speed for $25 \mathrm{~min}$ (third week), $70 \%$ of the maximum speed for $30 \mathrm{~min}$ (fourth to sixth weeks), and $40-50 \%$ of the a maximum speed for $5 \mathrm{~min}$ (cooldown). In the HIIT group, animals ran for $5 \mathrm{~min}$ at $40-50 \%$ of the maximum speed (warm-up). Each training consisted of high-intensity interval running ( $2 \mathrm{~min}$ ) and low-intensity running ( $2 \mathrm{~min}$ ) and was finished with a 5 -min running at $40-50 \%$ of the maximum speed (cooldown). The intensity for high-intensity interval running was at $80 \%$ of the maximum speed in the first week ( 5 intervals) and second week (6 intervals), $90 \%$ of the maximum speed in the third week ( 7 intervals) and $100 \%$ of the maximum speed in the fourth to sixth weeks (8 intervals). A maximal exercise capacity test was carried out every week, and the intensity of training was defined based on the new maximum speed each week. One day after the last training session, rats in the trained and control groups were tested by echocardiology [24], then all groups were sacrificed by intraperitoneal injection of ketamine ( $1 \mathrm{~g} / \mathrm{kg}$ body weight $)$ and xylazine $(10 \mathrm{mg} / \mathrm{kg})$, and next their lungs were extracted and analyzed by immunohistochemistry and real-time PCR.

\section{Immunohistochemistry}

Heart tissue samples were fixed in $4 \%$ formaldehyde in PBS and then rinsed with PBS. All samples were next dehydrated in ethanol and embedded in paraffin. For immunohistochemistry, $6 \mu \mathrm{m}$ paraffin-embedded sections were placed on adhesive plates and dried. Samples were also deparaffinized in xylene and rehydrated in alcohol series with decreasing concentrations. The EnVision System (Dako) was used to visualize the antigen-antibody reaction. All antibodies were diluted according to the manufacturer's instructions. The specimens were observed under an Olympus fluorescence microscope (Olympus, Tokyo, Japan) to detect the markers. Images were taken using a Canon Power Shot Camera.

\section{REAL-TIME PCR}

After the animals were sacrificed, total RNA was extracted from heart tissue samples by means of QIAzol ${ }^{\circledR}$ Lysis Reagent (Qiagen) according to the manufacturer's recommendations. RNA concentrations were determined by measuring absorbance at $260 \mathrm{~nm}$. RNA purity was also determined by the absorbance ratio at 260 and $280 \mathrm{~nm}$, and by ethidium bromide staining. An OD 260/ 280 ratio greater than 1.8 is usually considered an acceptable index for purified RNA. Afterwards, RNA was reverse transcribed into complementary DNA (cDNA) using a Revert Aid first standard cDNA Synthesis Kit (Thermo Scientific, Fermentas K1622, United States) using an accepted protocol including reverse transcription at $25^{\circ} \mathrm{C}$ for $5 \mathrm{~min}$, incubation with reverse transcriptase at $42{ }^{\circ} \mathrm{C}$ for $60 \mathrm{~min}$, and finally refrigeration at $70{ }^{\circ} \mathrm{C}$ for $5 \mathrm{~min}$, with storage at $-20{ }^{\circ} \mathrm{C}$.

For real-time PCR, primers were designed using NCBI and Gene Runner software, and they were synthesized by Cinnagen Company (Iran). The sequences of the primers are shown in Table 1 . The 
Table 1. The sequences of Nkx2.5 and GAPDH primers

\begin{tabular}{ll}
\hline Primer & \multicolumn{1}{c}{ Sequences } \\
\hline Nkx2-5 & FOR: 5' -CTTCAAGCAACAGCGGTACC-3' \\
& REV: 5'-ATCTTGACCTGCGTGGACG-3' \\
GAPDH & FOR: 5' - GACATGCCGCCTGGAGAAAC -3' \\
& REV: 5' - AGCCCAGGATGCCCTTTAGT -3' \\
\hline
\end{tabular}

measurement of gene expression was performed with Master Mix and SYBR Green by the Applied Biosystems, StepOne ${ }^{\mathrm{TM}}$ thermal cycler. The thermal cycle protocol was divided into the following protocols: 1 cycle at $95^{\circ} \mathrm{C}$ for $10 \mathrm{~min}$, followed by 40 cycles at $95^{\circ} \mathrm{C}$ for $15 \mathrm{~s}, 58^{\circ} \mathrm{C}$ for $30 \mathrm{~s}$, and $72{ }^{\circ} \mathrm{C}$ for $15 \mathrm{~s}$. PCR amplification was done in duplication in a total reaction volume of $20 \mu \mathrm{l}$. The reaction mixture consisted of $3 \mu \mathrm{l}$ diluted template, $10 \mu \mathrm{l}$ SYBR Premix Ex Taq ${ }^{\mathrm{TM}}$ Kit (Perfect Real Time, Takara Code RR041A, Japan), and $2 \mu$ l primers. Amplification specificity was monitored by the analysis of the melting curve. Genes' relative expressions were normalized by subtracting the housekeeping levels of glyceraldehyde-3-phosphate dehydrogenase $(\mathrm{GAPDH})$ mean $2^{-\Delta \Delta \mathrm{CT}}$, which was amplified as a housekeeping gene. All data are represented as fold-change in comparison to the control.

\section{Statistical analysis}

A Shapiro-Wilk test was conducted to confirm normal distribution of variables. One wayANOVA was used to evaluate the difference between groups. In the case of significance, LSD post hoc test was performed to determine differences between groups. Pearson correlation method was used to investigate the correlation between the degree of myogenesis and the intensity of exercise training. Results were reported as Mean \pm SD with the $\alpha$-level for significance set at 5\%. Data were analyzed using GraphPad Prism version 6.

\section{RESULTS}

The average of covered distance by the HIIT group was reported as $2.704 \mathrm{~m}$, whereas this value for the CET group was $2.830 \mathrm{~m}$, and the maximum speed of the rats in all three groups ranged from
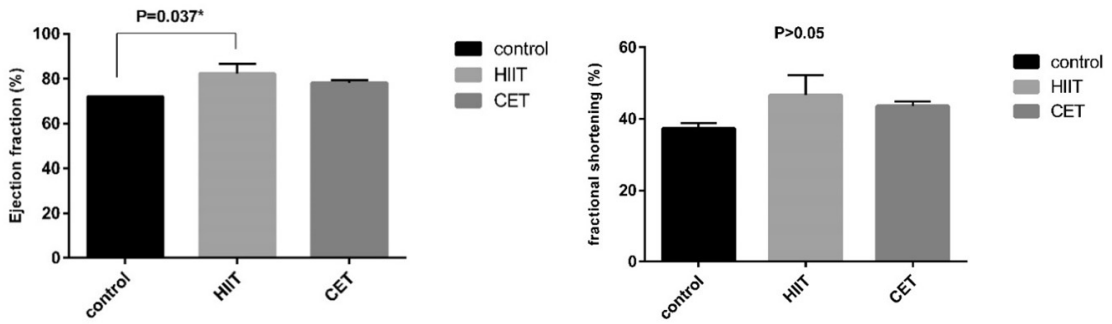

Figure 1. Ejection fraction and fractional shortening in the training group compared with the CO. The statistical analysis showed that the ejection fraction values $(\mathrm{HITT}=14.35 \%$, CET $8.79 \%)(P<0.05)$ were increased compared with the control group, but only the increase in the HIT group compared to the CO group was significant, and a non-significant increase was observed for fractional shortening (HIIT $=25 \%$, CET $16.96 \%)$ in both training groups compared to the control group. (Data are shown as mean + SD HIIT $* P<0.05$ vs. CO $P<0.05$ after 6 weeks) 
$32 \mathrm{~m} / \mathrm{min}$ in the pretest to $34 \mathrm{~m} / \mathrm{min}$ after 6 weeks of intervention. Significant differences were observed between the training groups and the control group for all variables. Echocardiography revealed that both types of training increased the ejection fraction, but the effect of HIIT was significantly greater in the aged rats. A non-significant increase was observed for fractional shortening in both training groups compared to the control group (Fig. 1). The results also showed
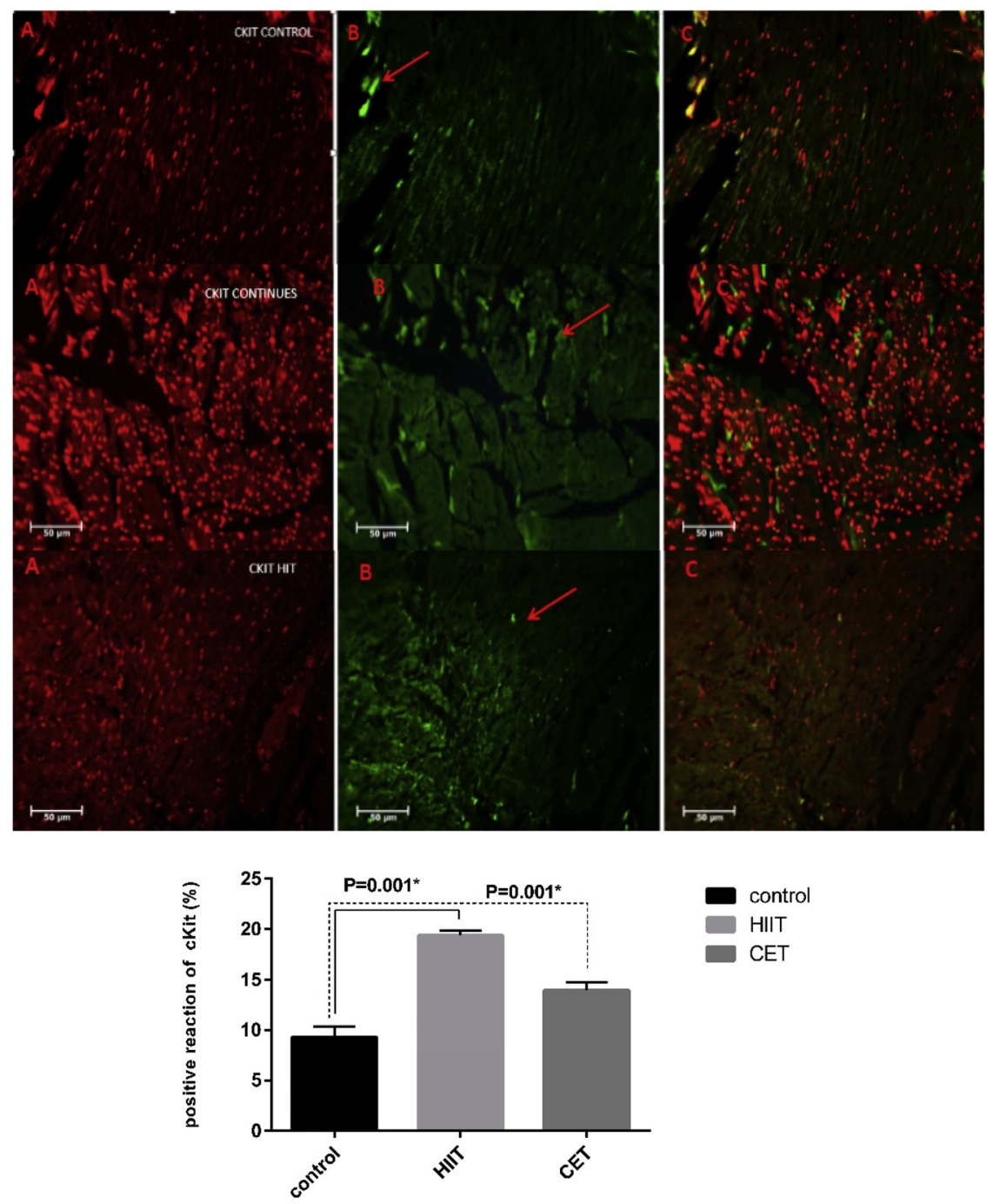

Figure 2. Representative image of C-kit showing that C-kit positive cells were increased in both training groups compared with the $\mathrm{CO}$ group. A higher rate of C-kit expression in cardiomyocytes was observed in the HIIT group that was dependent on the intensity of exercise. (All data are presented as the mean $+\mathrm{SD}, \mathrm{HIIT} * P<0.05$ and CET, ${ }^{*} P<0.05$ vs. CO $P<0.05$ after 6 weeks). Column A: nuclei were stained with propidium iodide (PI), column B: apoptosis-positive cells that have been shown by TUNEL assay (red arrows), column C: merge of A \& B 

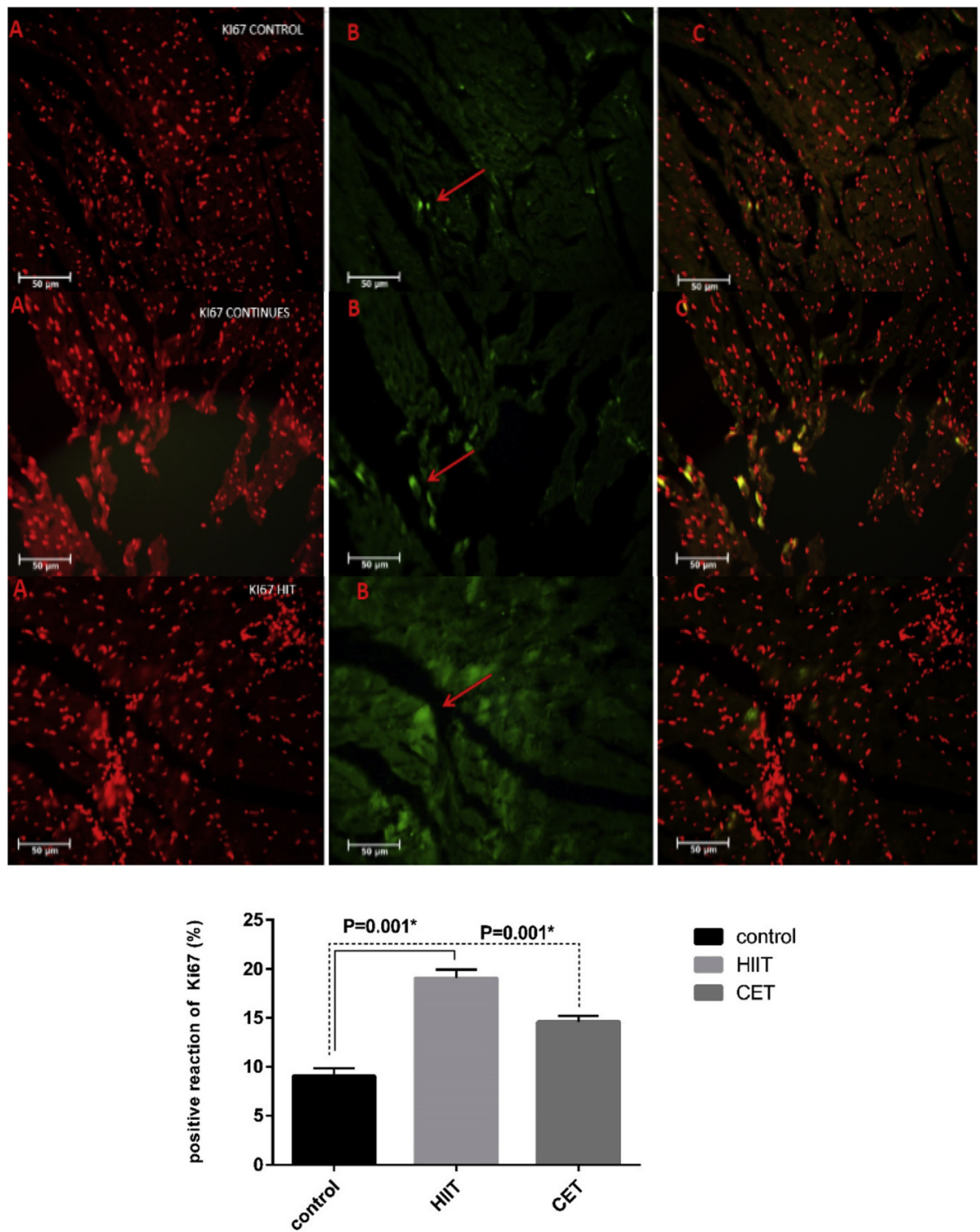

Figure 3. Representative images of small newly formed Ki67-positive (green) cardiomyocytes in both training groups. Some new myocyte formation was also detected in the $\mathrm{CO}$ group, but the number of cardiomyocytes was significantly lower than in the training groups. A higher number of Ki67-positive cardiomyocytes were observed in the HIIT group which was dependent on the exercise intensity (data are shown as the mean $+\mathrm{SD}, \mathrm{HIIT} * P<0.05$ and CET, ${ }^{*} P<0.05$ vs. CO $P<0.05$ after the 6 weeks). Column A: nuclei were stained with propidium iodide (PI), column B: apoptosis-positive cells that have been shown by TUNEL assay (red arrows), column C: merge of A \& B

that HIIT and CET increase cardiac myogenesis (Fig. 2). New cardiomyocyte formation (Ki67positive cells) was more extensive in the CET and HIIT groups, although its value was higher in the HIIT group after the 6-week training period as compared to CET (Fig. 3). 


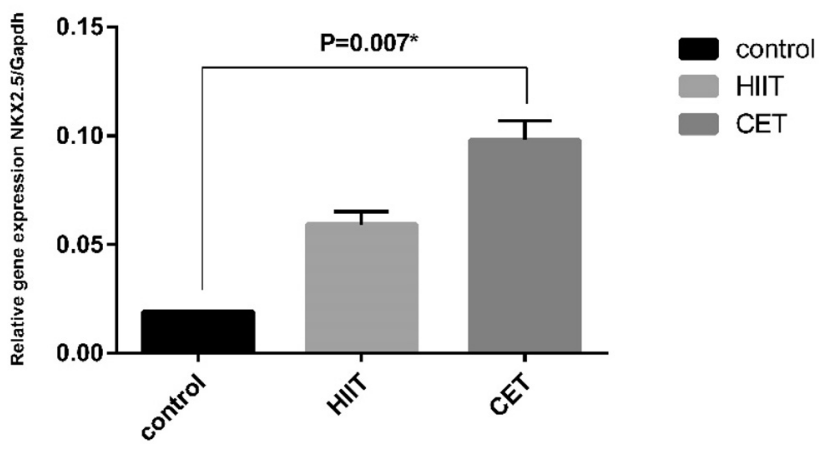

Figure 4. Exercise-induced changes in myocardial Nkx2.5 expression. RT-PCR analysis showed the upregulation of $\mathrm{Nkx} 2.5$ gene expression in the HIIT and CET groups compared with the CO group. Only the increase of $\mathrm{Nkx} 2.5$ expression in the CET group compared to the $\mathrm{CO}$ group was significant (data are shown as the mean $+\mathrm{SD}$, HIIT $P>0.05$ and CET, $* P<0.05$ vs. CO after 6 weeks)

To identify the source of the Ki67-positive cells as mentioned earlier, we determined the impact of exercise training on the activation and differentiation of the resident C-kit ${ }^{+}$(eCSCs).

The distribution of different types of eCSCs expressing cardiac lineage markers such as $\mathrm{Nkx} 2.5$ remained constant all over the study, suggesting that the level of activation is similar in these committed cells, leading to multiplication and differentiation (Fig. 4).

Finally, it seems that exercise training induces myocardial growth by stimulating the myogenesis. There was also a significantly higher rate of myogenesis in the training groups compared with the CO. The degree of myogenesis was positively correlated with the intensity of exercise training $(R=0.737, P=0.001)$.

\section{DISCUSSION}

The results of this study demonstrated that the physiological adaptation of the aged heart, induced by the exercise training program, has three main components: (i) physiological hypertrophy of the heart myocytes that was associated with the increased production of specific growth factors as well as the activation of C-kit-positive eCSCs; (ii) the activation of C-kitpositive eCSCs, which increase in number and undergo a process of cell specification and differentiation into myocytes; and [15]; (iii) the accumulation of new myocardial cells, namely new myocytes. These cellular modifications are dependent on the intensity of exercise and result in the enhancement of the cardiac function in aged rats.

\section{EFFECTS OF AGEING ON THE CARDIAC FUNCTION AND CSCs}

It is well-known that ageing is linked with the gradual decrease in physiological function and maintenance of hemostasis capacity that can lead to an increase in mortality and morbidity [18]. On the other hand, gradual decline in cardiac function during ageing has been shown. The increase of renewal capacity of myocytes is one of the important mechanisms improving cardiac 
function. However, it is unknown whether a defect in the growth and differentiation of cardiac stem cells is related to the ageing of cardiomyocytes and heart failure. It seems that ageingrelated factors may dramatically affect the growth and differentiation of CSCs $[3,5]$.

\section{MECHANISMS OF THE IMPROVEMENT OF CARDIAC FUNCTION IN AGEING THROUGH REGULAR EXERCISE AND THE EFFECTS OF EXERCISE TRAINING ON CARDIAC REGENERATION}

The beneficial effect of exercise is not limited to a mere reduction in the classical ageing-related cardiovascular risk factors, but it also influences the cellular and molecular structure and function of the aged heart [27]. In the present study, we demonstrated that exercise training improves neomyogenesis and facilitates the differentiation of the eCSCs into newly formed cardiomyocytes. The results also provide new insight into the molecular mechanisms underlying the regenerative effect of exercise on the aged heart. It seems that aged myocytes response to the increased workload through the upregulation of a sequence of secreted growth factors. The factors upregulated by extecise training differentially govern C-kit-positive CSCs. The niche of C-kit cells in the heart has key properties including multipotency, clonogenicity, and self-renewing $[5,17]$.

Thus, eCSCs existing in the heart exhibit a response to various growth factors that recapitulate, to some extent, embryonic cardiac progenitor activation and lineage commitment. Waring et al. showed that the adult heart responds to increased workload via physiological adaptation such as physiological hypertrophy, stem cell activation, and regeneration of new cardiomyocytes [25].

The current study indicated that exercise training for six weeks resulted in a significant increase in the expression of C-kit and the number of Ki67-positive cells in the heart of aged rats. The increase in C-kit expression and the number of Ki67-positive cells were both significantly higher in the HIIT group than in the CET group. An increase in the number of cells positive for Ki67 induced by the exercise training may, in part, represent the proliferation of myocytes. Hence, we can hypothesize that the increase in C-kit-positive CSCs could be due to an increase in the rate of proliferation of these cells or to a reduction in the apoptosis/necrosis rate as suggested by Waring et al. and Leite et al. [17, 25].

Also, the gene expression of Nkx2.5 was elevated in the HIIT and CET groups as compared with the control group. The increase in the expression of Nkx2.5 in the CET group was more extensive than in the HIIT group. It has been indicated that exercise training plays an important role in cell differentiation to regenerate cardiomyocytes as well as in the formation of new myocytes during ageing. The transcription factor Nkx2.5 is essential for the formation and development of the cardiac cells, and it is involved in cardiac hypertrophy. An increase in Nkx2.5 expression due to exercise training could lead to an increase in the differentiation of quiescent stem cells into cardiomyocytes [19].

Waring et al. showed that some physiological adaptation such as physiological hypertrophy, stem cell activation, and regeneration of new cardiomyocytes takes place in the adult heart in response to increased training intensity [25]. Xiao et al. demonstrated that 21 days of swimming significantly increases the levels of C-kit and Sca-1 in rats. Exercise training could cause physiological hypertrophy and activate progenitor cardiac stem cells [26]. Previous studies demonstrated that there is a direct correlation between the intensity of exercise and the changes in the number of newly formed cardiomyocytes in animal models and human studies $[4,24]$. 
In the present study, the 6-week aerobic exercise period improved the self-renewal capacity and cardiac function including fractional shortening and ejection fraction. However, high-intensity interval training induced a greater effect on cardiac function and self-renewal capacity. Waring et al. indicated that aerobic capacity, ejection fraction, fractional shortening, and anatomical indices of the cardiac tissue were considerably higher in the rats trained with low- and high-intensity exercise. It was shown that the HIIT group had a better cardiac function and structure in comparison with the low-intensity group [25]. Angadi et al. showed that $\mathrm{VO}_{2}$ peak and diastolic function were improved after 4 weeks of training in the HIIT group. It seems that the release of growth factor from myocytes stimulated by HIIT could lead to an increase in the number newly formed myocytes [2].

It seems that the formation of new cardiomyocytes in response to exercise training is caused by an increase in exercise-induced cardiomyocyte cell death. Cardiomyocyte cell death, which is thought to be induced by exercise stress, is a new hypothesis proposing that exercise-induced apoptosis can act as a key stimulus for the increase of cardiac self-regeneration [25]. According to previous studies, it also seems that an increase in growth factor due to exercise training is one of the possible mechanisms for cardiac self-regeneration, which improved the cardiac function in the HIIT and CET groups compared with the CO group in our study. Leite et al. reported that a 4week swimming period increases quiescent CSCs in the heart of rats and it is associated with exercise-induced physiological hypertrophy [17]. The present study indicated that exercise training in aged rats could increase myocytes' numbers and repair cardiac tissue in the heart of aged rats by stimulating quiescent CSCs $[11,25,26]$. Boström et al. reported that exercise training leads to a $6 \%$ increase in the percentage of cardiomyocyte regeneration in trained rats [8]. They believe newly formed myocytes in exercised animals originate from mitosis of the adult cardiomyocytes [25].

As a result, myocardial response to exercise training is dependent not only on the type of exercise training used, but rather it especially relies on the intensity at which the exercise is carried out. The present study showed that there is a strong relationship between training intensity and myogenesis, which indicates that training intensity has a greater effect than training type.

\section{CONCLUSION}

The present study showed that a 6-week aerobic exercise comprised of HIIT and CET is able to significantly increase the number of cardiac stem cells. It appears that the increase in cardiac stem cell numbers relates to the intensity of exercise. Based on our findings, we suggest that exercise training is capable of delaying or preventing the reduction in cardiac function and CSC number in the course of the ageing process. Accordingly, exercise training remarkably increases the self-regeneration capacity of cardiac cells in aged subjects. Even though we found that the number of C-kit-positive CSCs increases in response to exercise training and provided some explanations for the mechanism of exercise-induced cardiac cell improvement, other potential mechanisms cannot be ruled out, and further studies are needed to elucidate how exercise can stimulate regenation of the aged heart in senescence.

\section{REFERENCES}

1. Abel ED, Doenst T. Mitochondrial adaptations to physiological vs. pathological cardiac hypertrophy. Cardiovasc Res. 2011;90:234-42. 
2. Angadi SS, Mookadam F, Lee CD, Tucker WJ, Haykowsky MJ, Gaesser GA. High-intensity interval training vs. moderate-intensity continuous exercise training in heart failure with preserved ejection fraction: a pilot study. J Appl Physiol. 2014;119:753-58.

3. Anversa P, Kajstura J, Leri A, Bolli R. Life and death of cardiac stem cells. Circulation 2006;113:1451-63.

4. Beltrami AP, Barlucchi L, Torella D, Baker M, Limana F, Chimenti S, et al. Adult cardiac stem cells are multipotent and support myocardial regeneration. Cell 2003;114:763-76.

5. Bergmann O, Bhardwaj RD, Bernard S, Zdunek S, Barnabé-Heider F, Walsh S, et al. Evidence for cardiomyocyte renewal in humans. Science 2009;324:98-102.

6. Bergmann O, Jovinge S. Cardiac regeneration in vivo: mending the heart from within? Stem Cell Res. 2014;13: 523-31.

7. Bersell K, Arab S, Haring B, Kühn B. Neuregulin1/ErbB4 signaling induces cardiomyocyte proliferation and repair of heart injury. Cell 2009;138:257-70.

8. Boström P, Mann N, Wu J, Quintero PA, Plovie ER, Panáková D, et al. C/EBP $\beta$ controls exercise-induced cardiac growth and protects against pathological cardiac remodeling. Cell 2010;143:1072-83.

9. Buja LM, Vela D. Cardiomyocyte death and renewal in the normal and diseased heart. Cardiovasc Pathol. 2008;17:349-74.

10. Chimenti I, Gaetani R, Barile L, Forte E, Ionta V, Angelini F, et al. Isolation and expansion of adult cardiac stem/progenitor cells in the form of cardiospheres from human cardiac biopsies and murine hearts. Methods Mol Biol. 2012;879:327-38.

11. Ellison G, Mendicino I, Sacco W, Purushothaman C, Indolfi C, Goldspink D, et al. Exercise-induced cardiac stem cell activation and ensuing myocyte hyperplasia contribute to left ventricular remodelling. Proc Physiol Soc - Heart Cardiac Muscle Abstr. 2008;11:C17.

12. Foster C, Farland CV, Guidotti F, Harbin M, Roberts B, Schuette J, et al. The effects of high intensity interval training vs steady state training on aerobic and anaerobic capacity. J Sports Sci Med. 2015;14:747-55.

13. Gambini E, Pompilio G, Biondi A, Alamanni F, Capogrossi MC, Agrifoglio M, et al. C-kit+ cardiac progenitors exhibit mesenchymal markers and preferential cardiovascular commitment. Cardiovasc Res. 2010;89: $362-73$.

14. Hambrecht R, Wolf A, Gielen S, Linke A, Hofer J, Erbs S, et al. Effect of exercise on coronary endothelial function in patients with coronary artery disease. New England J Med. 2000:342;454-60.

15. Kerr III EL. An investigation of glycolysis, metabolic acidosis, and lactate's role in cellular respiration (Honors College Theses, 51). Wayne State University, DigitalCommons@WayneState; 2019.

16. Lakatta EG. So! What's aging? Is cardiovascular aging a disease?. J Mol Cell Cardiol. 2015;83:1-13.

17. Leite CF, Lopes CS, Alves AC, Fuzaro CSC, Silva MV, de Oliveira LF, et al. Endogenous resident c-Kit cardiac stem cells increase in mice with an exercise-induced, physiologically hypertrophied heart. Stem Cell Res. 2015;15:151-64.

18. Liu J, Rigel DF. Echocardiographic examination in rats and mice. Methods Mol Biol. 2009;573:139-55.

19. Ruan Z, Zhu L, Yin Y, Chen G. Overexpressing NKx2. 5 increases the differentiation of human umbilical cord drived mesenchymal stem cells into cardiomyocyte-like cells. Biomed Pharmacother. 2016;78:110-15.

20. Schlesinger J, Schueler M, Grunert M, Fischer JJ, Zhang Q, Krueger T, et al. The cardiac transcription network modulated by Gata4, Mef2a, Nkx2. 5, Srf, histone modifications, and microRNAs. PLoS Genet. 2011;7: e1001313.

21. Senyo SE, Steinhauser ML, Pizzimenti CL, Yang VK, Cai L, Wang M, et al. Mammalian heart renewal by preexisting cardiomyocytes. Nature. 2013;493:433-36.

22. Taddei S, Galetta F, Virdis A, Ghiadoni L, Salvetti G, Franzoni F, et al. Physical activity prevents age-related impairment in nitric oxide availability in elderly athletes. Circulation 2000;101:2896-901. 
23. Urbanek K, Torella D, Sheikh F, De Angelis A, Nurzynska D, Silvestri F, et al. Myocardial regeneration by activation of multipotent cardiac stem cells in ischemic heart failure. Proc Natl Acad Sci USA. 2005;102: 8692-97.

24. Van Laake LW, Hassink R, Doevendans PA, Mummery C. Heart repair and stem cells. J Physiol. 2006;577: 467-78.

25. Waring CD, Vicinanza C, Papalamprou A, Smith AJ, Purushothaman S, Goldspink DF, et al. The adult heart responds to increased workload with physiologic hypertrophy, cardiac stem cell activation, and new myocyte formation. Eur Heart J. 2014;35:2722-31.

26. Xiao J, Xu T, Li J, Lv D, Chen P, Zhou Q, et al. Exercise-induced physiological hypertrophy initiates activation of cardiac progenitor cells. Int J Clin Exp Pathol. 2014;7:663-69.

27. Yan L, Gao S, Ho D, Park M, Ge H, Wang C, et al. Calorie restriction can reverse, as well as prevent, aging cardiomyopathy. Age 2013;35:2177-82.

28. Yang L, Soonpaa MH, Adler ED, Roepke TK, Kattman SJ, Kennedy M, et al. Human cardiovascular progenitor cells develop from a KDR+ embryonic-stem-cell-derived population. Nature 2008;453:524-28. 